\title{
Approche Lagrangienne des écoulements diphasiques multidimensionnels
}

\author{
par Alexandre Hulin, Elie Znaty \\ de la Société Bertin
}

\section{I 瞌 APPROCHE LAGRANGIENNE ET ÉQUA- TIONS DU MOUVEMENT}

L'idée de base de l'approche lagrangienne est de suivre les trajectoires des particules en résolvant les équations de mouvement. Cette approche consiste en 3 étapes:

- les équations eulériennes représentant la phase continue sont résolues,

- l'effet de la phase continue sur les particules et sur leurs trajectoires est calculé,

- la rétroaction des particules sur la phase continue est évaluée (même si cette rétroaction est assez souvent négligée).

Il faut insister sur le fait que ces interactions dépendent du diamètre des particules. En particulier, les particules de petite taille, dont les tailles sont de l'ordre de grandeur des tourbillons, dissipent l'énergie turbulente alors que les grosses particules pompent l'énergie cinétique et créent des tourbillons et des sillages turbulents. Autrement dit, les petites particules font décroître l'énergie turbulente alors que les grosses ont tendance à l'augmenter.

Le mouvement d'une particule sphérique peut être décrit par l'équation suivante :

$$
\frac{\pi d^{3}}{6} \rho_{p} \frac{\mathrm{d} u p}{\mathrm{~d} t}=F
$$

La force $\mathrm{F}$ contient deux types de contributions:

- la force qu'il y aurait si l'écoulement était non perturbé (forces de pression et hydrostatique),

- la force due à l'interaction avec le fluide (frottement, forces de Basset, masse ajoutée,...).

On démontre que pour $\rho<<p$, ( $p$ pour particule) les deux seuls termes résiduels sont la gravité et le frottement visqueux. L'équation du mouvement s'écrit donc:

$$
F_{p}=\frac{1}{8} C_{D} \mu \pi d^{2}\left|u-u_{p}\right|\left(u-u_{p}\right)+\frac{\pi d^{3}}{6}\left(\rho_{p}-\rho\right) g
$$

Mathematical modelling of two phase flows gained profits from progress made in computational fluid dynamics and from increased needs in availability of simulation tools for processes involving multiphasic mixing. Two approaches, called eulerian and lagrangian, have been proposed for two phase flow modelling.

Lagrangian approach is described with a focuse on physical models for mass, momentum, internal energy, turbulent kinetic energy exchanges. Coupling between dispersed phase and continuous phase is described as well.

In order to show that the two approaches, generally claimed to be in competition, are complementary, specific ranges of applications of each method are presented. Some results showing evaporation and two way coupling between dispersed phase and continuous phase turbulence are briefly described.

As a conclusion, possible evolutions of two phase flow modelling are sketched In particular some thoughts on the coupling between lagrangian and eulerian approaches are presented. Also, specific aspects related to parallelisation of an eulerian code using lagrangian approach for the dispersed phase are outlined. 


$$
=\rho_{p} \frac{\pi d^{3}}{6} \frac{\left(u-u_{p}\right)}{\tau_{p}}+\frac{\pi d^{3}}{6}\left(\rho_{p}-\rho\right) g
$$

avec $\tau_{p}=\frac{\rho_{p}}{\rho} \frac{4 d}{3 C_{l}} \frac{1}{\left|u-u_{p}\right|}$

avec $\tau_{p}$ le temps de relaxation

$C_{d}$ le coefficient de trainée fonction du nombre de Reynolds.

Cette équation du mouvement fait l'hypothèse que le temps de relaxation $\tau_{p}$ est grand devant le temps caractéristique $\tau_{v}=\frac{\rho d^{2}}{\mu}$.

De même, la taille caractéristique de la particule $d$ doit être petite devant la taille caractéristique de variation du champ de vitesse.

Ainsi, sous ces hypothèses, on doit résoudre l'équation du mouvement. Malgré son apparente simplicité, elle pose plusieurs problèmes:

- $u$ la vitesse du fluide intervenant dans l'équation est la vitesse instantanée du fluide ( $1^{\text {re }}$ difficulté) au lieu de la particule $\left(2^{\circ}\right.$ difficulté),

- $u_{p}$ est la vitesse instantanée de la particule.

Or, les modélisations ne donnent accès (sauf pour la DNS) qu'à des valeurs moyennes alors qu'on a besoin des valeurs instantanées. Deux étapes doivent être analysées quand on veut déterminer la vitesse instantanée de la particule à un instant $t+\Delta t$ quand on connaît la vitesse instantanée de la particule et la fluctuation de vitesse à un instant $t$ où la particule et le paquet fluide sont corrélés:

- il faut être capable de déterminer la fluctuation de vitesse du paquet fluide à l'instant $t+\Delta t$ (attention, le paquet fluide initialement corrélé avec la particule n'est pas au même endroit que la particule à linstant $t+\Delta t$ ),

- il faut être capable de déterminer la fluctuation de vitesse du fluide autour de la particule à l'instant $t+\Delta t$.

\section{MODÈLE EULÉRIEN POUR LA PHASE PORTEUSE}

La phase continue est modélisée en utilisant la moyenne de Favre dans les équations de Navier Stokes pour des écoulements turbulents et compressibles. On utilise le système d'équations pour la masse volumique $\bar{\rho}$, la vitesse moyenne $\tilde{u}$ et l'énergie interne $\tilde{e}$ :

$$
\begin{aligned}
& \frac{\partial}{\partial t} \bar{\rho}+\nabla(\bar{\rho} \tilde{u})=\bar{S}_{p}^{d} \\
& \frac{\partial}{\partial t} \bar{\rho} \tilde{u}+\nabla(\bar{\rho} \tilde{u} \tilde{u}+\bar{\sigma})=\bar{S}_{\rho \mu}^{d} \\
& \frac{\partial}{\partial t} \bar{\rho} \tilde{e}+\nabla(\bar{\rho} \tilde{u} \tilde{e})+\bar{\sigma} \nabla u+\nabla \bar{J}=\bar{D}_{k}-\nabla \bar{\phi}_{k}+\bar{S}_{p e}^{d}
\end{aligned}
$$

où $\bar{\sigma}=\bar{\sigma}^{l}+\bar{\sigma}^{\prime}$

$$
\begin{aligned}
& \bar{\sigma}^{\prime}=\bar{p} I d+\bar{\tau}^{\prime}=\bar{p} I d-\mu^{\prime}\left[\nabla \tilde{u}+\nabla \tilde{u}^{\prime}-\frac{2}{3} \nabla \tilde{u} I d\right] \\
& \bar{\sigma}^{\prime}=\frac{2}{3} p \bar{k} l d-\mu^{\prime}\left[\nabla \tilde{u}+\nabla \tilde{u}^{\prime}-\frac{2}{3} \nabla \tilde{u} I d\right]=\frac{2}{3} \bar{\rho} \bar{k} l d+\bar{\tau}^{\prime}
\end{aligned}
$$

$\tilde{k}=\frac{1}{2} r\left(u^{\pi} u^{\prime \prime}\right)$

$\bar{J}=\bar{J}^{\prime}+\bar{J}^{t}$

$\vec{J}^{\prime}=-k^{\prime} \nabla \bar{T}$

$\bar{J}^{t}=-k^{\prime} \nabla \bar{T}$ et $k^{\prime}=\mu^{\prime} \frac{C_{p}}{P^{\prime}}$

$\bar{\sigma}$ le tenseur de contrainte est décomposé en une partie sphérique $\bar{\rho}+\frac{2}{3} \bar{\rho} \tilde{k}$ et une partie déviatorique contenant une contribution laminaire et une contribution turbulente.

De la même façon, le flux de chaleur contient une partie laminaire et une partie turbulente modélisée grâce à l'analogie de Reynolds par la définition d'un nombre de Prandtl turbulent. On peut remarquer que les flux d'énergie dus aux flux de diffusion d'espèces sont négligés. La modélisation de la turbulence est effectuée en utilisant un modèle à 2 équations: $\bar{k}$ l'énergie cinétique turbulente et $\tilde{\varepsilon}$ son taux de dissipation :

$$
\begin{aligned}
& \frac{\partial}{\partial t} \bar{\rho} \tilde{k}+\nabla\left(\bar{\rho} \tilde{u} \tilde{k}+\bar{\phi}_{k}\right)=\bar{P}_{k}+\bar{D}_{k}+\bar{S}_{p k}^{l} \\
& \frac{\partial}{\partial t} \bar{\rho} \varepsilon+\nabla\left(\bar{\rho} \tilde{u} \tilde{\varepsilon}+\bar{\phi}_{\varepsilon}\right)=\frac{\tilde{\varepsilon}}{\bar{k}}\left(C_{z: 1} \bar{P}_{k}+C_{k: 2} \bar{D}_{k}\right)+\bar{S}_{\rho \varepsilon}^{d}
\end{aligned}
$$

avec $\bar{P}_{k}$ la production de $\bar{k}, \bar{D}_{k}$ la destruction de $\bar{k}, \bar{\phi}_{k}\left(\bar{\phi}_{z}\right)$ le flux de diffusion de $\hat{k}(\tilde{\varepsilon})$

$$
\begin{aligned}
& \bar{P}_{k}=-\bar{\sigma}^{\prime} \nabla \tilde{u} \\
& \bar{D}_{k}=-\bar{\rho} \tilde{e} \\
& \bar{\phi}_{s}=-\left(\mu^{\prime}+\frac{\mu^{\prime}}{P r^{*}}\right) \nabla \tilde{s} \text { pour } \tilde{s} \text { égal à } \tilde{k} \text { ou } \tilde{\varepsilon}
\end{aligned}
$$$$
\text { et } \quad \mu^{\prime}=\bar{\rho} C_{\mu} \frac{\tilde{k}^{2}}{\tilde{\varepsilon}}
$$

Les échanges entre la phase continue et la phase dispersée sont représentés par les termes source $S^{d}$ d'origine diphasique (indice haut):

$$
\begin{array}{ll}
\bar{S}_{p}^{l} & \text { échange de masse, } \\
\bar{S}_{p l d}^{l} & \text { échange de quantité de mouvement, } \\
\bar{S}_{p e}^{l} & \text { échange d'énergie, } \\
\bar{S}_{p k}^{l} \text { et } \bar{S}_{p \varepsilon}^{d} \text { contribution à la turbulence de phase conti- } \\
\text { nue. }
\end{array}
$$

Pour calculer ces termes source, il est nécessaire de représenter la phase dispersée.

\section{MODÈLE LAGRANGIEN POUR LA PHASE DISPERSÉE}

Le modèle lagrangien consiste à résoudre pour chaque particule un système d'équations pour la masse $m_{p}$, pour la vitesse $u_{p}$, la position, la température $T_{p}$, de cette particule. 


\subsection{Echange de masse}

L'échange de masse est basé sur deux niveaux :

- détermination de l'échange de masse d'une goutte supposée sphérique au repos avec hypothèse d'équilibre thermodynamique liquide vapeur à la surface de la goutte,

- correction de Ranz Marshall pour tenir compte de la convection en introduisant un nombre de Nusselt :

$$
\begin{gathered}
m_{p}=m_{p \text { mepos }} N_{u} \\
N_{u}=2+0.6 R_{u}^{1 / 2} p_{r}^{1 / 3}
\end{gathered}
$$

L'échange de masse au repos dépend du régime:

\section{- Evaporation :}

Le gaz environnant n'est pas saturé en phase gazeuse du liquide évaporant $\left(Y_{\text {vapur }}<1\right)$; dans ce cas, le taux d'évaporation $\dot{m}$, est piloté par la diffusion et la loi de Clapeyron.

$$
\dot{m}_{\text {pripos }}=\pi d \frac{k}{C p_{p}} \log (1+B)
$$

avec $C p_{1}$, la capacité calorifique de la vapeur du liquide évaporant et $B$ le nombre de soufflage.

\section{- Ebullition :}

Le gaz enviromnant est saturé en vapeur et dans ce cas, le taux d'évaporation $\dot{m}_{p}$ est piloté par l'échange thermique conductif entre le gaz et le liquide, et la température du liquide est bloquée à la température de vapeur saturante.

\subsection{Echange de quantité de mouvement}

Le coefficient de traînée intervenant dans l'équation du mouvement $C_{D}$ est classiquement exprimé par des formules empiriques dépendant du nombre de Reynolds basé sur la taille de la particule et le glissement de vitesse gazparticule :

$$
\begin{array}{ll}
C_{D}=\frac{24}{R_{c p}} & \text { pour } R_{c p}<20 \\
C_{D}=\frac{24}{R_{c p}}\left(1+0.1315 R_{c p}^{0.82}-1.533\right) & \text { pour } 20<R_{e p p}<110 \\
C_{D}=\frac{24}{R_{c p}}\left(1+0.185 R_{c p}^{0.687}\right) & \text { pour } 110<R_{c p}<1000 \\
\text { où } R_{c p}=\rho \frac{\left|u-u_{p}\right| d}{\mu} &
\end{array}
$$

\subsection{Echange de chaleur}

Le modèle de transfert d'énergie tient compte des échanges convectifs entre le gaz et la particule ainsi que des apports d'énergie pour les changements de phase (évaporation du liquide).

Ainsi, le bilan d'énergie pour la goutte peut s'écrire sous la forme:

$$
\begin{aligned}
& m_{p} C_{p} \frac{d}{\mathrm{~d} t} T_{p}=\dot{q}_{c(m)}+\dot{q}_{m a t s \mathrm{se}} \\
& \operatorname{avec} \dot{q}_{c o n}=h S p\left(T-T_{p}\right)=\frac{\lambda N u}{d} S p\left(T-T_{p}\right) \\
& \dot{q}_{m a t: s e}=-\dot{m}_{p} h_{\text {sadp }}
\end{aligned}
$$

où Sp est la surface d'échange de la particule.

\subsection{Reconstitution de la vitesse vue par la particule}

La vitesse de la phase continue vue par la particule est composée de deux contributions: $u=\tilde{u}+u^{\prime \prime}$. La vitesse moyenne est évaluée par interpolation linéaire dans chaque direction au droit de la particule du champ de vitesse moyen calculé sur le maillage eulérien. Cette interpolation nécessite la localisation de la particule dans le maillage eulérien.

La vitesse instantanée est reconstituée de façon qu'en moyenne elle soit égale à $\sqrt{\frac{2}{3}} \bar{k}$. Ainsi, on prend: $u^{\prime \prime}=\xi\left(\frac{2}{3} \tilde{k}\right)^{1 / 2}$ avec $\xi$ un vecteur de 3 variables aléatoires tirées à partir d'une loi normale (gaussienne).

\subsection{Effet de la turbulence sur la particule}

L'effet de la turbulence sur la phase dispersée apparaît à 2 niveaux:

- la détermination de la vitesse instantanée vue par la particule,

- le temps caractéristique $\tau_{p,}$ apparaissant dans l'échange de quantité de mouvement entre particule et fluide.

Le temps caractéristique $\tau_{p}$ doit tenir compte de deux phénomènes, à savoir la durée de vie moyenne des tourbillons $\tau_{p}$ et le temps de traversée du tourbillon par la particule $\tau_{s}$. Le modèle de base est de considérer qu'à un instant donné, une particule et un paquet fluide (un tourbillon) sont corrélés; on s'intéresse alors au temps au bout duquel la particule est décorrélée d'avec ce tourbillon.

On pose : $\tau_{y}=2 \eta \frac{l}{\sqrt{k}}$

$$
\tau_{s}=\frac{l}{\left|u-u_{p}\right|}
$$

où

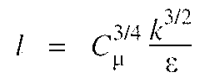

est une taille caractéristique du tourbillon

$\eta \quad$ une variable aléatoire uniforme (le facteur 2 provient de ce que la moyenne de $\eta$ est $1 / 2$ )

Enfin, on pose $\tau_{p}$ tel que $\frac{1}{\tau_{p}}=\frac{1}{\tau_{p}}+\frac{1}{\tau_{s}}$

\subsection{Questions non résolues}

Ce modèle d'interaction entre la turbulence et la phase dispersée nous a conduit à effectuer des choix non nécessairement justifiés :

- La vitesse moyenne $\tilde{u}$ vue par la particule est réactualisée à chaque pas de temps alors que la fluctuation de vitesse $u^{\prime \prime} n$ 'est réactualisée qu'après que l'horloge interne 
de chaque particule ait atteint le temps d'interaction $\tau_{p}, \mathrm{Ce}$ choix fait l'hypothèse que le paquet fluide avec lequel la particule est corrélée réagit instantanément aux variations de la vitesse moyenne et que la vitesse fluctuante reste constante pendant le temps d'interaction.

- Nous avons affecté la durée de vie moyenne $\tau_{f}$ d'une variable aléatoire uniforme $\eta$ de façon à ne pas avoir une dépendance par rapport au pas de temps de calcul du champ eulérien, mais nous avons considéré que le temps de traversée $\tau_{s}$ est déterministe.

- Le temps de traversée $\tau_{s}$ du tourbillon par la particule est donné en fonction de la vitesse de glissement et de la taille caractéristique du tourbillon. Toutefois, si le modèle considère qu à l'instant initial, la particule est corrélée avec le centre du tourbillon, il faudrait considérer comme distance à parcourir par la particule pour se décorréler la moitié de la taille caractéristique.

- L'effet de remplissage maximal $\left(\alpha_{\text {disp }} \leqslant 1\right)$ n'est pas représenté. Une possibilité serait d'introduire la correction dite d'Ergun à activer pour $\alpha_{\text {disp }}>0.1$.

\subsection{Modèle de couplage}

L'objectif du modèle de couplage est de modéliser les termes source apparaissant dans les seconds membres des bilans de masse, de quantité de mouvement, d'énergie, d'énergie cinétique turbulente et de son taux de dissipation. Ce modèle de couplage consiste à sommer dans chaque cellule la contribution de chaque particule présente dans cette maille.

Il est clair que, compte tenu du nombre réel de particules, on choisit pour des raisons de coût, de représenter par une seule particule "numérique » un certain nombre de particules réelles. Ainsi, chaque terme source peut s'écrire sous la forme:

$$
\bar{S}\left(\Omega_{n e l}\right)=\sum_{p \in \Omega_{w a l}} \bar{s}(p) P(p)
$$

où $\Omega_{n e l}$ est le volume de la maille nel,

$\bar{s}(p)$ est la contribution de la particule $p$,

$P(p)$ est le poids numérique de chaque particule $p$.

De cette façon, on peut exprimer tous les termes source déjà introduits :

$$
\begin{aligned}
& \bar{S}_{p}^{d}\left(\Omega_{n e l}\right)=\frac{1}{\operatorname{vol}\left(\Omega_{n e l}\right)} \sum_{p \in \Omega_{w l}} \dot{m}_{p} P(p) \\
& \bar{S}_{p u}^{d}\left(\Omega_{n e l}\right)=\frac{1}{v o l\left(\Omega_{n e l}\right)} \sum_{p \in \Omega_{u e l}} \\
& \left\{-m_{p} F_{p}+m_{p}\left(u_{p}-\tilde{u}\right)\right\} P(p) \\
& \bar{S}_{p e}^{l l}\left(\Omega_{n e l}\right)=\frac{1}{\operatorname{vol}\left(\Omega_{n e l}\right)} \sum_{p \in \Omega_{n c l}}\left\{\dot{q}_{c(m,}+\dot{q}_{\text {massse }}\right\} P(p) \\
& \bar{S}_{p k}^{d}\left(\Omega_{n e l}\right)=\frac{1}{\operatorname{vol}\left(\Omega_{n e l}\right)} \sum_{p \in \Omega_{w e t}} \\
& \left\{-m_{p} F_{p}\left(\tilde{u}-u_{p}\right)-m_{p}\left(\tilde{u}-u_{p}\right)^{2}\right\} P(p) \\
& \bar{S}_{p \varepsilon}^{l}\left(\Omega_{n e l}\right)=C_{i} \frac{\tilde{\varepsilon}}{\bar{k}} S_{p k}^{d}\left(\Omega_{n e l}\right)
\end{aligned}
$$

Le terme source de quantité de mouvement fait les hypothèses suivantes:

- la vitesse d'éjection de vapeur est négligée,

- la poussée d'Archimède est négligée.

Le terme source de quantité de mouvement est valable en instationnaire et est composé de deux termes: la réaction des forces de trânée et la contribution due au gaz injecté par changement de phase.

Le terme source apparaissant dans l'équation de l'énergie cinétique turbulente est calculé par différence entre la moyenne de l'équation de quantité de mouvement instantanée multiplié par la vitesse instantanée, et l'équation de quantité de mouvement moyennée multipliée par la vitesse moyenne :

$$
\bar{S}_{p h}^{d l}\left(\Omega_{n e l}\right)=\overline{u S_{p u}^{d}}\left(\Omega_{n e l}\right)-\tilde{u} \bar{S}_{p u}^{d l}\left(\Omega_{n e l}\right)
$$

\section{ANALYSE DE LA COMPLÉMENTA- RITÉ DES APPROCHES EULÉRIENNE ET LAGRANGIENNE}

Les deux approches eulérienne et lagrangienne permettent d'utiliser les mêmes modèles physiques pour l'échange de masse, le frottement, l'échange d'énergie.

Le modèle lagrangien est bien adapté à la modélisation des écoulements dispersés, car il ne contient pas la représentation de l'effet de la phase dispersée sur le champ de pression.

Le modèle lagrangien permet de bien représenter des milieux hétérogènes car il permet de ne calculer des trajectoires que là où il y a des particules.

De plus, il est possible d'améliorer la représentativité de l'échantillon de particules de chaque taille dans chaque maille. De même, il est possible d'ajuster l'échantillonnage à la précision souhaitée. Ces deux ajustements sont rendus possibles en jouant sur le nombre de particules physiques que représente chaque particule calculée.

Par ailleurs, l'approche lagrangienne permet une bonne reconstitution du couplage entre les phénomènes physiques intervenant dans la phase continue et la phase dispersée. Il apparait donc clairement un besoin d'extension et un besoin de validation de l'approche lagrangienne pour les milieux denses.

En parallèle, l'approche eulérienne à deux fluides consiste à écrire un système d'équation de bilan de masse, quantité de mouvement et éneigie pour la phase continue, et un système pour la phase dispersée. Cette approche est bien adaptée au traitement des milieux denses car elle permet un couplage fort entre les deux phases. Non seulement, on peut introduire les termes d'échange entre phases, l'effet de la turbulence de la phase continue sur la phase dispersée et la rétroaction de la phase dispersée sur la turbulence de la phase continue.

Elle présente néanmoins un inconvénient : c'est le traitement de la distribution de taille de particules. En effet, à moins d'écrire un système pour chaque taille de particules, il est difficile en pratique d'avoir plus qu'une taille moyenne de particules. 


\section{QUELQUES RÉSULTATS}

Des jets sous-détendus, chargés en gouttelettes liquides présentent un phénomène dit de flashing. Lorsque le jet se développe, une forte détente crée une chute de pression suffisante pour évaporer brutalement le liquide présent sous forme de gouttes. Ce phénomène crée un apport important de masse de gaz même lorsque la densité initiale en gouttes est faible. Une expérience numérique a été réalisée avec un jet d'ammoniac chargé en gouttes de $2 \mu \mathrm{m}$. La densité initiale en particules est $10^{-4}$. Il apparaît nettement sur les cartes iso-taux volumique de phase dispersée (fig. l) que la majorité des particules s'évaporent toutes en sortie de buse d'éjection, dans la zone supersonique à basse pression. Ce phénomène se retrouve sur l'évolution en temps du diamètre des particules (fig. 2) et sur les cartes d'isoconcentration en fraction massique d'ammoniac gazeux (fig. 3).

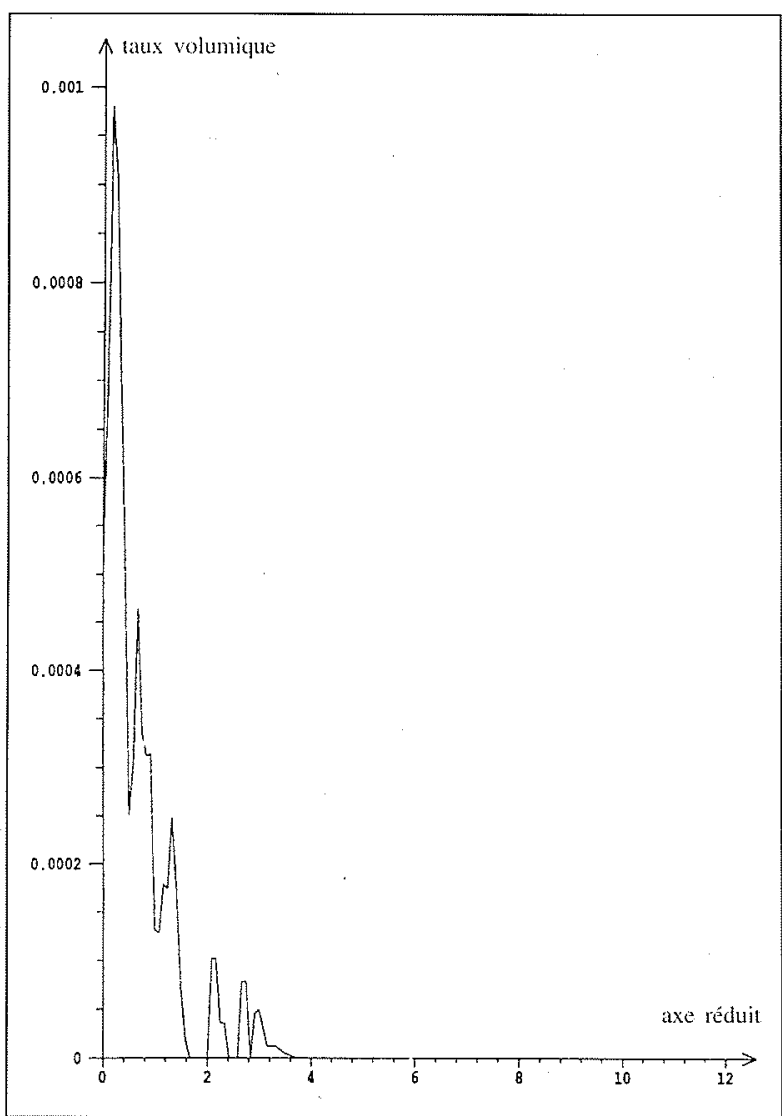

1. Taux volumique de gouttes dans un jet d'ammoniac Evolution sur l'axe du jet.

\section{PERSPECTIVES}

\subsection{Parallélisation de l'approche lagrangienne}

Deux méthodes pour la parallélisation de l'approche lagrangienne sont candidates:

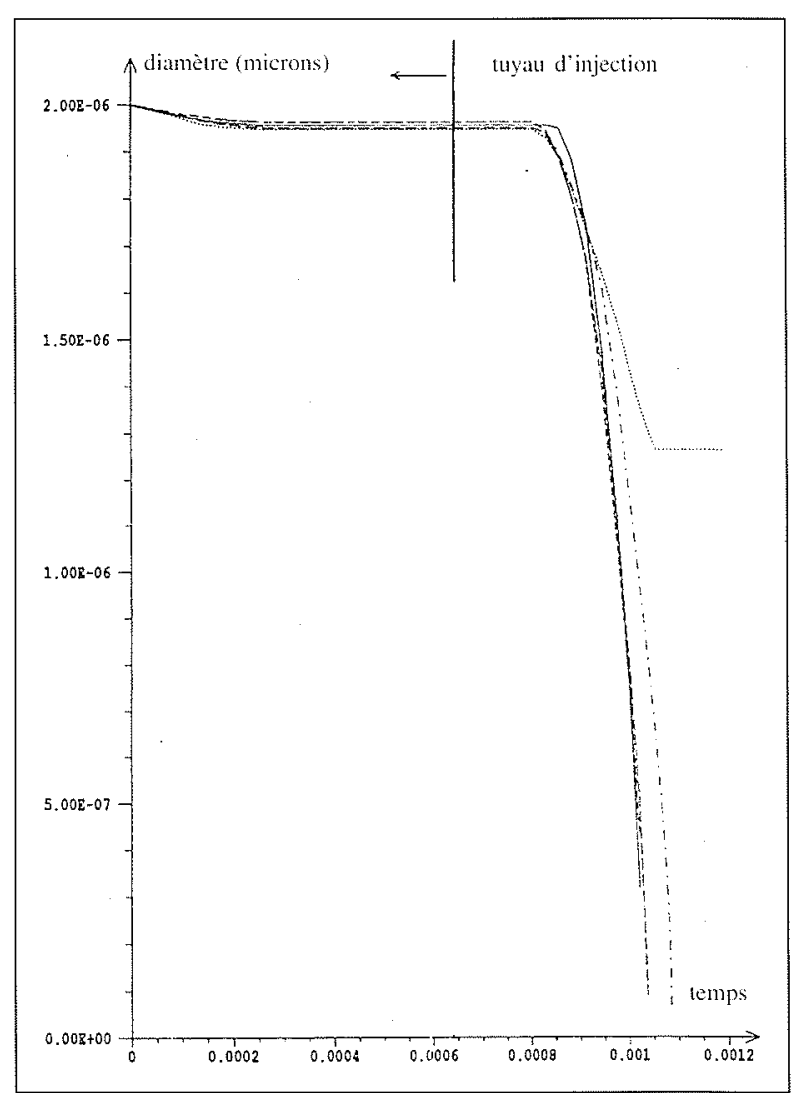

2. Evolution du diamètre.

\section{- Première méthode :}

Elle consiste à décomposer le domaine en sous-domaines et à affecter le traitement d'un sous-domaine à un processus de calcul. Le processus qui traite un sous-domaine doit donc traiter les particules qui s'y trouvent. Cette méthode présente l'avantage d'être cohérente avec l'idée de parallélisation dite SPMD (Single Program Multiple Data) dans le sens que le même programme est distribué sur tous les processus. Elle nécessite de savoir traiter le cas des gouttes qui changent de sous-domaine.

Elle présente l'inconvénient de ne pas garantir une distribution de tâche bien équilibrée entre les processus car rien ne garantit que chaque sous-domaine contiendra un nombre équivalent de particules.

\section{- Deuxième méthode :}

La deuxième méthode consiste à distribuer les particules sur les processus de calcul indépendamment de leur localisation dans le maillage eulérien. Cette approche nécessite la mise en place et l'utilisation d'un scénario de communication dit «all-to-all»: chaque processus doit être capable de communiquer avec n'importe quel autre processus. De plus, il faut pouvoir localiser une particule dont on connaît les coordonnées dans un maillage distribué, car en effet, il n'est pas envisageable de dupliquer la totalité du maillage dans chaque processus.

Nous avons choisi la première approche. Ainsi la difficulté majeure liée au modèle diphasique est la localisation 


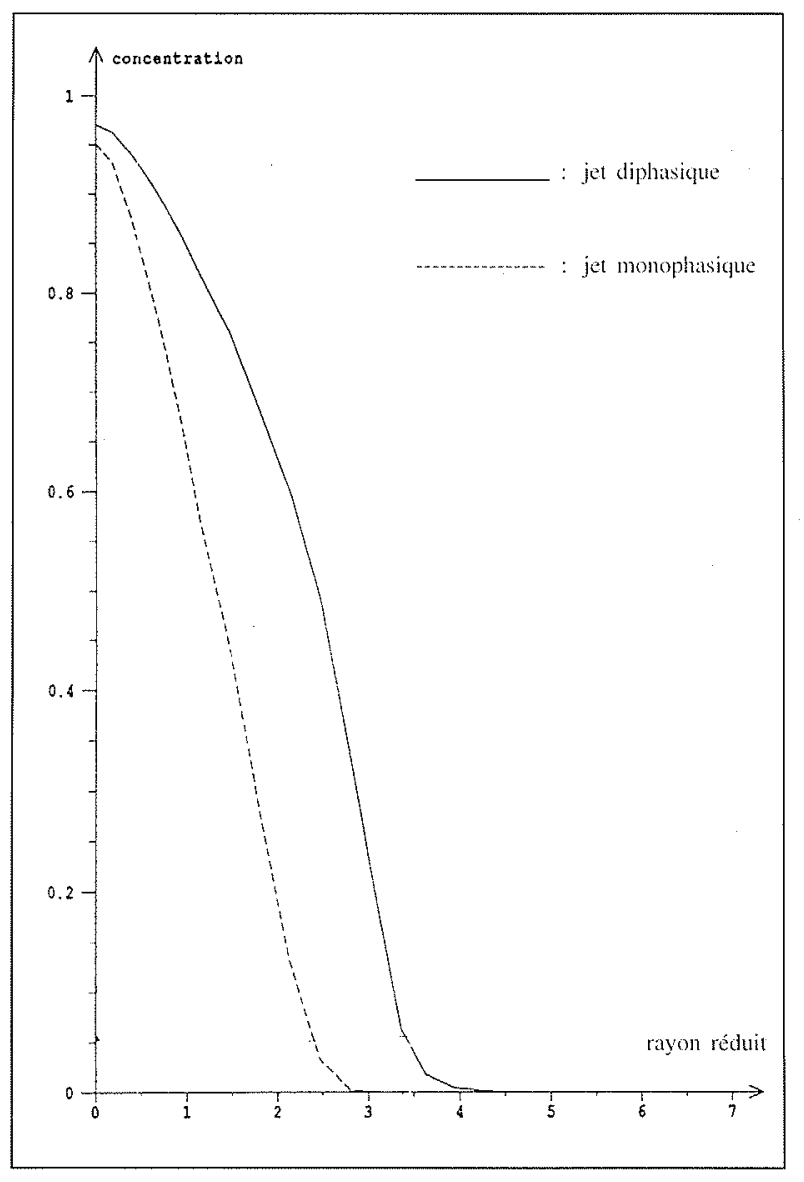

3. Fraction molaire d'ammoniac gazeux. Coupe transverse à 15 diamètres.

des particules qui ont «quitté » le sous-domaine auquel elles appartiennent. L'algorithme de localisation peut être écrit en pseudolangage comme suit :

Pour tous les domaines voisins du domaine courant, faire :

Pour toutes les particules «perdues » par le domaine courant, faire :

envoyer les informations relatives à cette particule

essayer de localiser dans le domaine voisin courant la particule reçue

si localisée : alors la particule est trouvée

fin faire

Pour toutes les particules «perdues » par le domaine courant, faire:

libérer la place dans la structure de données fin faire
6.2 Quelques réflexions sur le couplage des deux approches lagrangienne et eulérienne

Une approche proposée pour pouvoir traiter une distribution de taille de particules en eulérien pourrait consister à :

- écrire un système eulérien à deux fluides,

- transporter en lagrangien une distribution de taille,

- calculer les termes d'échange par moyenne des termes source lagrangien et introduction dans le système eulérien à 2 fluides,

- calculer les termes source pour les équations de surface d'échange et de taux volumique de la phase dispersée par moyenne des densités de présence des particules et de leur surface par le calcul des trajectoires.

En fait, cette approche peut être vue comme une approche lagrangienne dans laquelle le processus de sommation de la contribution de chaque particule à chaque maille est remplacé par l'alimentation d'un système d'équations transport-diffusion avec terme source représentant la phase dispersée par les contributions de chaque particule. Ainsi, le système d'équations de bilan pour la phase dispersée apparaît comme une boitte de transfert des termes source d'origine diphasique. De même, cette approche peut être vue comme une approche eulérienne à deux fluides avec le remplacement de l'équation pour le taux de vide (ou pour le nombre densité de particules) par un transport lagrangien des particules et reconstitution des grandeurs moyennes nécessaires : volume occupé par les particules, surface d'échange,...

\section{CONCLUSION}

Une approche lagrangienne pour la modélisation des écoulements diphasiques multidimensionnels a été détaillée. La complémentarité entre les approches lagrangienne et eulérienne à 2 fluides est soulignée. Quelques perspectives d'évolution de cette approche sont présentées. Quelques questions non résolues sont aussi posées afin d'ouvrir le débat sur l'amélioration des modélisations des écoulements diphasiques.

\section{Remerciements}

Cette synthèse a été réalisée dans le cadre de la contri bution de la Société Bertin at Groupement de Recherche pour les Ecoulements Diphasigues en Conduite (GREDIC) finance par le MRES, le CNRS et EDF, et BERTIN pour la partie autofinancee).

Les auteurs profitent de cette synthèse pour remercier Monsieur le Professeur Delhaye pour ses actions nenees dans la coordination et l'animation du GREDIC. 